\title{
Coherently Driven $N$ Number of Degenerate Three-Level Atoms with Parametric Amplifier
}

\author{
Tamirat Abebe $(\mathbb{D}$, Chimdessa Gashu $(\mathbb{D}$, and Nebiyu Gemechu \\ Department of Physics, Jimma University, P. O. Box 378, Jimma, Ethiopia \\ Correspondence should be addressed to Tamirat Abebe; tam1704@gmail.com
}

Received 23 May 2020; Accepted 22 September 2020; Published 12 October 2020

Academic Editor: Yu-Hao Sun

Copyright (@ 2020 Tamirat Abebe et al. This is an open access article distributed under the Creative Commons Attribution License, which permits unrestricted use, distribution, and reproduction in any medium, provided the original work is properly cited.

\begin{abstract}
We have analyzed the squeezing and statistical properties of the cavity light beam produced by a coherently driven degenerate three-level laser with a degenerate parametric amplifier (DPA) in an open cavity and coupled to a vacuum reservoir via a singleport mirror. We have carried out our analysis by putting the noise operators associated with the vacuum reservoir in normal order. Applying the solutions of the equations of evolution for the expectation values of the atomic operators and the quantum Langevin equation for the cavity mode operator, the mean photon number and the quadrature squeezing of the cavity light are calculated. And a large part of the mean photon number is confined in a relatively small frequency interval. Furthermore, we also obtain the antinormally ordered characteristic function defined in the Heisenberg picture. With the aid of the resulting characteristic function, we determine the $Q$ function which is then used to calculate the photon number distribution.
\end{abstract}

\section{Introduction}

Three-level cascade lasers have received considerable interest in connection with its potential as a source of light with interesting nonclassical features [1-22]. The quantum properties of the light, in this device, are attributed to atomic coherence that can be induced either by preparing the atoms initially in a coherent superposition of the top and bottom levels $[3,12,13,22]$ or coupling these levels by an external radiation $[7,8,10]$ or using these mechanisms together [23].

Moreover, some authors have studied quantum properties of light generated by the three-level laser whose cavity contains parametric amplifier $[10,11]$. Parametric amplifier involves three different modes of the radiation fields the signal, the idler, and the pump which are coupled by a nonlinear medium. In this device a pump photon interacts with a nonlinear crystal inside a cavity and is down converted into two highly correlated photons of different frequencies [12]. These works indicated the cavity radiation is found to be in a squeezed and entangled states under certain conditions. In addition, the mean and variance of the photon number for a degenerate $[11,12,17]$ and nondegenerate [13-16] three- level cascade laser whose cavity contains parametric amplifier have been determined for different cases.

In the paper, we wish to study the squeezing and statistical properties of the light generated by a degenerate parametric amplifier (DPA) and coherently driven degenerate threelevel laser with an open cavity coupled to a vacuum reservoir via a single-port mirror. We carry out our calculation by putting the noise operators associated with the vacuum reservoir in normal order $[6,8,24,25]$. We thus first determine the master equation for a coherently driven degenerate threelevel laser in an open cavity coupled to a vacuum reservoir and the quantum Langevin equation for the cavity mode operator. In addition, employing the master equation and the large-time approximation scheme, we obtain equations of evolution of the expectation values of atomic operators. Moreover, we determine the solutions of the equations of evolution of the expectation values of the atomic operators and the quantum Langevin equation for cavity mode operator. Then, applying the resulting solutions, we calculate the photon statistics and the quadrature squeezing of the twomode light beams. Furthermore, applying the same solutions, we determine the antinormally ordered characteristic 
function defined in the Heisenberg picture [17, 24, 26]. With the aid of the resulting characteristic function, we determine the $Q$ function which is then used to calculate the photon number distribution.

\section{The Model}

We consider a coherently pumped degenerate three-level laser with DPA coupled to a vacuum reservoir whose cavity contains $N$ three-level atoms in cascade configuration as shown in Figure 1. The interaction of a degenerate threelevel atom with the cavity mode a can be described by the Hamiltonian.

$$
\widehat{H}_{1}=i g\left[\left(\widehat{\sigma}_{a}^{\dagger k}+\widehat{\sigma}_{b}^{\dagger k}\right) \widehat{a}-a \wedge^{\dagger}\left(\widehat{\sigma}_{a}^{k}+\widehat{\sigma}_{b}^{k}\right)\right]
$$

where $g$ is the coupling constant between the atom and cavity mode $a$, and $\hat{a}\left(a \wedge^{\dagger}\right)$ are the annihilation (creation) operators for cavity light mode. We also define that $\Omega=2 \mu \lambda$, in which $\mu$ considered to be real and constant, is the amplitude of the driving coherent light, and $\lambda$ is the coupling constant between the driving coherent light and the three-level atom. We also define that

$$
\begin{aligned}
& \widehat{\sigma}_{a}^{k}=|b\rangle_{k k}\langle a|, \\
& \hat{\sigma}_{b}^{k}=|c\rangle_{k k}\langle b|,
\end{aligned}
$$

are lowering atomic operators.

On the other hand, a pump mode photon of frequency, $\omega^{\prime}=2 \omega$, directly interacts with a parametric amplifier (DPA) to produce two signal photons having the same frequency. Furthermore, we consider the case for which the pump mode emerging from a DPA does not couple the top and bottom levels. This could be realized by putting on the right-hand side of the DPA a screen which absorbs the pump mode. With the pump mode treated classically, the process of a single-mode subharmonic generation can be described by the Hamiltonian $[22,26]$.

$$
\widehat{H}_{2}=\frac{i \varepsilon}{2}\left[a \wedge^{2}-a \wedge^{\dagger 2}\right]
$$

in which $\varepsilon=2 \beta \lambda_{0}$ with $\lambda_{0}$ is the coupling constant between the pump mode and nonlinear crystal, and $\beta$ is proportional to the amplitude of the coherent light driving pump mode. The coupling of the top and bottom levels of a three-level atom by coherent light can be described by the Hamiltonian $[7,8,24]$.

$$
\widehat{H}_{3}=\frac{i \Omega}{2}\left[\widehat{\sigma}_{c}^{\dagger k}-\widehat{\sigma}_{c}^{k}\right]
$$

Here, $\Omega=2 \mu \lambda_{1}$ where $\mu$, considered to be real and constant, is the amplitude of the driving coherent light, and $\lambda_{1}$ is the coupling constant between the driving coherent light with the three-level atom and $\widehat{\sigma}_{c}^{k}=|c\rangle_{k k}\langle a|$. Thus, upon combining Eqs. (1), (4), and (5), we have

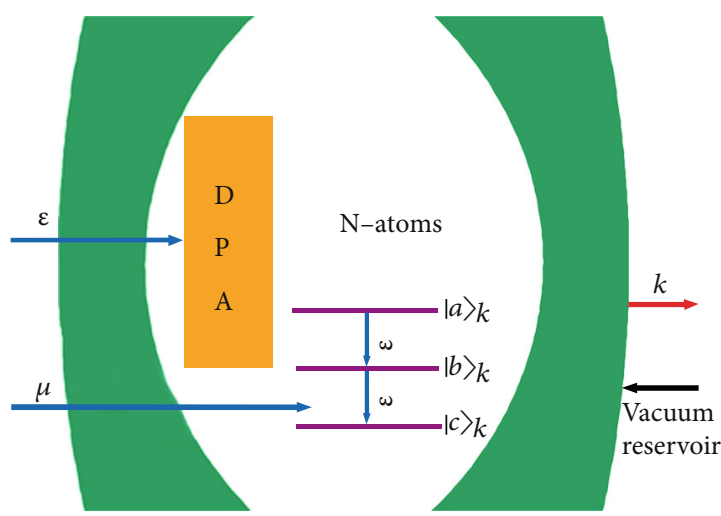

FIGURE 1: Schematic representation of a degenerate three-level laser with a degenerate parametric amplifier (DPA) and coupled to a twomode vacuum reservoir. Here, $\varepsilon$, considered to be real and constant, is proportional to the amplitude of the pump mode that drives the nonlinear crystal (NLC), and $\mu$ is the amplitude of the driving coherent light that couples the top and bottom levels of the atom. And also $\kappa$ is the cavity damping constant, and it is assumed the same for both transitions. The top, middle, and bottom levels of the three-level atom are denoted by $|a\rangle_{k},|b\rangle_{k}$, and $|c\rangle_{k}$, respectively, where as $k=1,2, \cdots N$ are the number of atoms inside the cavity. When the atom makes a transition from level $|a\rangle_{k} \rightarrow$ $\left\langle\left. b\right|_{k} \text { and from levels } \mid b\right\rangle_{k} \rightarrow\left\langle\left. c\right|_{k}\right.$ two photons with the same frequencies, $\omega$, are emitted.

$$
\begin{aligned}
\widehat{H}= & i g\left[\left(\widehat{\sigma}_{a}^{\dagger k}+\widehat{\sigma}_{b}^{\dagger k}\right) \widehat{a}-a \wedge^{\dagger}\left(\widehat{\sigma}_{a}^{k}+\widehat{\sigma}_{b}^{k}\right)\right] \\
& +\frac{i \varepsilon}{2}\left[a \wedge^{2}-a \wedge^{\dagger 2}\right]+\frac{i \Omega}{2}\left[\widehat{\sigma}_{c}^{\dagger k}-\widehat{\sigma}_{c}^{k}\right] .
\end{aligned}
$$

In addition, the master equation for a coherently driven degenerate three-level laser with a one-mode subharmonic generator coupled to a vacuum reservoir in an open cavity, following the procedure described in Ref. $[7,24]$, is found to be

$$
\begin{aligned}
\frac{d}{d t} \widehat{\rho}(t)= & -i\left[\widehat{H}_{S}, \widehat{\rho}(t)\right]+\frac{\gamma}{2}\left[2 \widehat{\sigma}_{a}^{k} \widehat{\rho} \widehat{\sigma}_{a}^{\dagger k}-\widehat{\sigma}_{a}^{\dagger k} \widehat{\sigma}_{a}^{k} \widehat{\rho}-\widehat{\rho} \widehat{\sigma}_{a}^{\dagger k} \widehat{\sigma}_{a}^{k}\right] \\
& +\frac{\gamma}{2}\left[2 \widehat{\sigma}_{b}^{k} \widehat{\rho} \widehat{\sigma}_{b}^{\dagger k}-\widehat{\sigma}_{b}^{\dagger k} \widehat{\sigma}_{b}^{k} \widehat{\rho}-\widehat{\rho} \widehat{\sigma}_{b}^{\dagger k} \widehat{\sigma}_{b}^{k}\right]
\end{aligned}
$$

where $\gamma$, considered to be the same for all the three levels, is the spontaneous emission decay constant. In view of Eq. (6), Eq. (7) turns out to be

$$
\begin{aligned}
\frac{d}{d t} \widehat{\rho}(t)= & g\left[\widehat{\sigma}_{a}^{\dagger k} \widehat{a} \widehat{\rho}-a \wedge^{\dagger} \widehat{\sigma}_{a}^{k} \widehat{\rho}+\widehat{\sigma}_{b}^{\dagger k} \widehat{a} \widehat{\rho}-a \wedge^{\dagger} \widehat{\sigma}_{b}^{k} \widehat{\rho}-\widehat{\rho} \widehat{\sigma}_{a}^{\dagger k} \widehat{a}\right. \\
& \left.+\widehat{\rho} a \wedge^{\dagger} \widehat{\sigma}_{a}^{k}-\widehat{\rho} \widehat{\sigma}_{b}^{\dagger k} \widehat{a}+\widehat{\rho} a \wedge^{\dagger} \widehat{\sigma}_{b}^{k}\right] \\
& +\frac{\gamma}{2}\left[2 \widehat{\sigma}_{a}^{k} \widehat{\rho} \widehat{\sigma}_{a}^{\dagger k}-\widehat{\sigma}_{a}^{\dagger k} \widehat{\sigma}_{a}^{k} \widehat{\rho}-\widehat{\rho} \widehat{\sigma}_{a}^{\dagger k} \widehat{\sigma}_{a}^{k}\right] \\
& +\frac{\gamma}{2}\left[2 \widehat{\sigma}_{b}^{k} \widehat{\rho} \widehat{\sigma}_{b}^{\dagger k}-\widehat{\sigma}_{b}^{\dagger k} \widehat{\sigma}_{b}^{k} \widehat{\rho}-\widehat{\rho} \widehat{\sigma}_{b}^{\dagger k} \widehat{\sigma}_{b}^{k}\right] \\
& +\frac{\varepsilon}{2}\left[a \wedge^{2} \widehat{\rho}-\widehat{\rho} a \wedge^{2}+\widehat{\rho} a \wedge^{\dagger 2}-a \wedge^{\dagger 2} \widehat{\rho}\right] \\
& +\frac{\Omega}{2}\left[\widehat{\sigma}_{c}^{\dagger k} \widehat{\rho}-\widehat{\sigma}_{c}^{k} \widehat{\rho}+\widehat{\rho} \widehat{\sigma}_{c}^{\dagger k}-\widehat{\rho} \widehat{\sigma}_{c}^{k}\right] .
\end{aligned}
$$


We recall that the laser cavity is coupled to a single-mode vacuum reservoir via a single-port mirror. In addition, we carry out our calculation by putting the noise operators associated with the vacuum reservoir in normal order. Thus, the noise operators will not have any effect on the dynamics of the cavity mode operators $[7,8]$. We can therefore drop the noise operators and write the quantum Langevin equations for the operator $\hat{a}$ as

$$
\frac{d \widehat{a}}{d t}=-\frac{\kappa}{2} \widehat{a}-i[\widehat{a}, \widehat{H}]
$$

where $\kappa$ is the cavity damping constant. Then, in view of Eq. (6), we see that

$$
\frac{d \widehat{a}}{d t}=-\frac{\kappa}{2} \widehat{a}-g\left(\widehat{\sigma}_{a}^{k}+\widehat{\sigma}_{b}^{k}\right)-\varepsilon a \wedge^{\dagger} .
$$

We see that Eqs. (A.4)-(A.8) are nonlinear differential equations and hence it is not possible to find exact timedependent solutions of these equations. We intend to overcome this problem by applying the large-time approximation $[7,8,24,25,27]$. Therefore, employing this approximation scheme, we get from Eq. (10) the approximately valid relation.

$$
\widehat{a}=-\frac{2 g}{\kappa}\left(\widehat{\sigma}_{a}^{k}+\widehat{\sigma}_{b}^{k}\right)-\frac{2 \varepsilon}{\kappa} a \wedge^{\dagger} .
$$

Evidently, this turns out to be exact relation at steady state. Employing the adjoint of this equation into Eq.(10), one easily verify that

$$
\frac{d \widehat{a}}{d t}=-\frac{1}{2}\left(\frac{\kappa^{2}-4 \varepsilon^{2}}{\kappa}\right) \widehat{a}-g\left(\widehat{\sigma}_{a}^{k}+\widehat{\sigma}_{b}^{k}\right)-\frac{2 \varepsilon g}{\kappa}\left(\widehat{\sigma}_{a}^{\dagger k}+\widehat{\sigma}_{b}^{\dagger k}\right) .
$$

The solution of this equation is

$$
\widehat{a}=-\frac{4 \varepsilon g}{\kappa^{2}-4 \varepsilon^{2}}\left(\widehat{\sigma}_{a}^{k}+\widehat{\sigma}_{b}^{k}\right)-\frac{2 g \kappa}{\kappa^{2}-4 \varepsilon^{2}}\left(\widehat{\sigma}_{a}^{\dagger k}+\widehat{\sigma}_{b}^{\dagger k}\right) .
$$

In the presence of $N$ three-level atoms, we rewrite Eq. (12) as

$$
\frac{d \widehat{a}}{d \mathrm{t}}=-\frac{1}{2}\left(\frac{\kappa^{2}-4 \varepsilon^{2}}{\kappa}\right) \widehat{a}+\lambda^{\prime}\left(\widehat{m}_{a}+\widehat{m}_{b}\right)+\lambda^{\prime \prime}\left(\widehat{m}_{a}^{\dagger}+\widehat{m}_{b}^{\dagger}\right) .
$$

in which $\lambda^{\prime}$ and $\lambda^{\prime \prime}$ are constants whose values remain to be fixed. Then, using the definition $\widehat{m}=\widehat{m}_{a}+\widehat{m}_{b}$ [27], we obtain

$$
\frac{d \widehat{a}}{d t}=-\frac{1}{2}\left(\frac{\kappa^{2}-4 \varepsilon^{2}}{\kappa}\right) \widehat{a}+\lambda^{\prime} \widehat{m}+\lambda^{\prime \prime} m \wedge^{\dagger} .
$$

Moreover, employing Eq. (13), the commutation relations of the cavity mode operators are

$$
\left[\widehat{a}, a \Lambda^{\dagger}\right]_{k}=\frac{\gamma_{c} \kappa}{\kappa^{2}-4 \varepsilon^{2}}\left[\kappa^{2}\left[\widehat{\eta}_{c}^{k}-\widehat{\eta}_{a}^{k}\right]+4 \varepsilon^{2}\left[\widehat{\eta}_{a}^{k}-\widehat{\eta}_{c}^{k}\right]\right],
$$

and on summing over all atoms, we have

$$
\left[\widehat{a}, a \Lambda^{\dagger}\right]=\frac{\gamma_{c} \mathcal{K}}{\kappa^{2}-4 \varepsilon^{2}}\left[\kappa^{2}\left[\widehat{N}_{c}-\widehat{N}_{a}\right]+4 \varepsilon^{2}\left[\widehat{N}_{a}-\widehat{N}_{c}\right]\right],
$$

where $\left[\widehat{a}, a \Lambda^{\dagger}\right]=\sum_{k=1}^{N}\left[\widehat{a}, a \Lambda^{\dagger}\right]_{k}$. We note that Eq. (17) stands for the commutators $\widehat{a}$ and $a \wedge^{\dagger}$ when the cavity mode is interacting with all the $N$ three-level atoms. On the other hand, using the steady-state solution of Eq. (15), one can easily verify that

$$
\left[\widehat{a}, a \wedge^{\dagger}\right]=\frac{4 \lambda^{\prime 2} \kappa^{2} N}{\left(\kappa^{2}-4 \varepsilon^{2}\right)}\left[\widehat{N}_{c}-\widehat{N}_{a}\right]+\frac{4 \lambda^{\prime \prime 2} \kappa^{2} N}{\left(\kappa^{2}-4 \varepsilon^{2}\right)}\left[\widehat{N}_{a}-\widehat{N}_{c}\right] .
$$

Upon comparing Eqs. (17) and (18), one can see that

$$
\lambda^{\prime}= \pm \frac{g}{\sqrt{N}}, \lambda^{\prime \prime}= \pm \frac{2 g \varepsilon}{\kappa \sqrt{N}} .
$$

Hence, in view of these results, the equation of evolution of the cavity mode operator given by Eq. (15) can be written as

$$
\frac{d \widehat{a}}{d t}=-\frac{1}{2}\left(\frac{\kappa^{2}-4 \varepsilon^{2}}{\kappa}\right) \widehat{a}+\frac{g}{\sqrt{N}} \widehat{m}+\frac{2 g \varepsilon}{\kappa \sqrt{N}} m \wedge^{\dagger} .
$$

The steady-state solution of Eq. (20) is

$$
\widehat{a}=\frac{2 g \kappa}{\sqrt{N}\left(\kappa^{2}-4 \varepsilon^{2}\right)} \widehat{m}+\frac{4 g \varepsilon}{\sqrt{N}\left(\kappa^{2}-4 \varepsilon^{2}\right)} m \Lambda^{\dagger} .
$$

The expectation value of the solution the annihilation operator for the cavity mode evolves in time according to the quantum Langevin equation given by Eq. (20) can be expressed as

$$
\begin{aligned}
\langle\widehat{a}(t)\rangle= & \langle\widehat{a}(0)\rangle e^{-\eta t / 2}+\frac{g}{\sqrt{N}} e^{-\eta t / 2} \int_{0}^{t} e^{\eta t^{\prime} / 2}\left\langle\widehat{m}\left(t^{\prime}\right)\right\rangle d t^{\prime} \\
& +\frac{2 g \varepsilon}{\kappa \sqrt{N}} e^{-\eta t / 2} \int_{0}^{t} e^{\eta t^{\prime} / 2}\left\langle m \Lambda^{\dagger}\left(t^{\prime}\right)\right\rangle d t^{\prime},
\end{aligned}
$$

where $\eta=\left(\kappa^{2}-4 \varepsilon^{2}\right) / \kappa$. Thus, applying the large-time approximation scheme, we find the adjoint solution of Eq. (A.10) to be

$$
\left\langle\widehat{m}_{b}^{\dagger}(t)\right\rangle=-\frac{\zeta_{+}}{v}\left\langle\widehat{m}_{a}(t)\right\rangle
$$

Now, on substituting Eq. (23) into Eq. (A.9), it is not difficult to see that

$$
\frac{d}{d t}\left\langle\widehat{m}_{a}(t)\right\rangle=-\frac{1}{2} \eta_{0}\left\langle\widehat{m}_{a}(t)\right\rangle,
$$

where $\eta_{0}=\left(2 v^{2}-\zeta_{-} \zeta_{+}\right) / v$. With the atoms considered to be initially in the bottom level, the solution of Eq. (24) is found to be 


$$
\left\langle\widehat{m}_{a}(t)\right\rangle=\left\langle\widehat{m}_{b}(t)\right\rangle=0 .
$$

In view of these results together with $\widehat{m}=\widehat{m}_{a}+\widehat{m}_{b}$, one can see that

$$
\langle\widehat{m}(t)\rangle=0 \text {. }
$$

With the help of Eq. (26) and the assumption that the cavity light is initially in a vacuum state, Eq. (22) turns out to be

$$
\langle\widehat{a}(t)\rangle=0
$$

In view of the linear equation described by Eq. (20) along with (27), we claim that $\hat{a}(t)$ is a Gaussian variable with zero mean. Finally, the steady-state solutions of the atomic operators are found to be

$$
\begin{aligned}
\left\langle\widehat{m}_{c}\right\rangle_{\mathrm{ss}} & =\left[\frac{2 v(\xi+\Omega)}{4 v^{2}+2 \Gamma(2 \xi+\Omega)+(2 \Omega+\xi) \zeta_{+}}\right] N, \\
\left\langle\widehat{N}_{a}\right\rangle_{\mathrm{ss}} & =\left[\frac{\zeta_{+}(\xi+\Omega)}{4 v^{2}+2 \Gamma(2 \xi+\Omega)+(2 \Omega+\xi) \zeta_{+}}\right] N, \\
\left\langle\widehat{N}_{b}\right\rangle_{\mathrm{ss}} & =\left[\frac{2 \Gamma v(\xi+\Omega)}{4 v^{2}+2 \Gamma(2 \xi+\Omega)+(2 \Omega+\xi) \zeta_{+}}\right] N, \\
\left\langle\widehat{N}_{c}\right\rangle_{\mathrm{ss}} & =N-\frac{\left(\zeta_{+}+2 \Gamma v\right)(\xi+\Omega) N}{4 v^{2}+2 \Gamma(2 \xi+\Omega)+(2 \Omega+\xi) \zeta_{+}}
\end{aligned}
$$

where ss stands for steady state and

$$
\Gamma=\frac{\zeta_{+}(v-\gamma)-4 \xi v}{2 v^{2}}
$$

\section{Quadrature Squeezing}

The squeezing properties of the cavity light are described by two quadrature operators defined by

$$
\begin{gathered}
\widehat{a}_{+}=a \wedge^{\dagger}+\widehat{a}, \\
\widehat{a}_{-}=i\left(a \wedge^{\dagger}-\widehat{a}\right),
\end{gathered}
$$

where $\widehat{a}_{+}$and $\hat{a}_{-}$are Hermitian operators representing the physical quantities called plus and minus quadrature. With the aid of Eqs. (33) and (34) along with (21), the two quadrature operators satisfy the commutation relation

$$
\left[\widehat{a}_{-}, \widehat{a}_{+}\right]=2 i \frac{\gamma_{c}}{k}\left[\frac{\kappa^{2}}{\kappa^{2}-4 \varepsilon^{2}}\right]\left[\widehat{N}_{a}-\widehat{N}_{c}\right]
$$

An interesting application of the commutator algebra is to derive a relation giving the uncertainties product of two operators $\widehat{A}$ and $\widehat{B}$, i.e, $\Delta A \Delta B \geq 1 / 2|\langle[\widehat{A}, \widehat{B}]\rangle|$. Hence, in view of this, the uncertainty relation of the two quadrature operators can be written as

$$
\Delta a_{+} \Delta a_{-} \geq \frac{\gamma_{c}}{k}\left[\frac{\kappa^{2}}{\kappa^{2}-4 \varepsilon^{2}}\right]\left|\left\langle\widehat{N}_{a}\right\rangle-\left\langle\widehat{N}_{c}\right\rangle\right|
$$

On account of Eqs. (29) and (31), Eq. (36) takes the form $\Delta a_{+} \Delta a_{-} \geq \frac{\gamma_{c}}{k}\left[\frac{\kappa^{2}}{\kappa^{2}-4 \varepsilon^{2}}\right] N \times\left|1-\frac{\left(\zeta_{+}+\xi+2 \Gamma v\right)(\xi+\Omega)}{4 v^{2}+2 \Gamma(2 \xi+\Omega)+(2 \Omega+\xi) \zeta_{+}}\right|$

It is interesting to consider some special cases. We first inspect the case in which the nonlinear crystal is removed from the cavity. Thus, upon setting $\beta=0$ in Eq. (37), we get

$$
\Delta a_{+} \Delta a_{-} \geq \frac{\gamma_{c}}{\kappa} N\left|\frac{\delta^{3}}{\delta^{3}+\left(\gamma_{c}+2 \delta\right) \Omega^{2}}\right|,
$$

where $\delta=\gamma+\gamma_{c}$.

In addition, we consider the case in which the top and bottom levels of the atoms do not coupled by the pump mode. Hence, upon setting $\Omega=0$ in Eq. (38), we see that

$$
\Delta a_{+} \Delta a_{-} \geq \frac{\gamma_{c}}{k} N
$$

which is the minimum uncertainty relation for vacuum state.

Next, we proceed to calculate the quadrature variance of the cavity light. The variance of the plus and minus quadrature operators of the cavity light are defined by

$$
\Delta a_{ \pm}^{2}=\left\langle\widehat{a}_{ \pm}^{2}\right\rangle-\left\langle a \wedge_{ \pm}\right\rangle^{2} .
$$

It then follows that

$$
\Delta a_{ \pm}^{2}=\left\langle\widehat{a} a \wedge^{\dagger}\right\rangle+\left\langle a \wedge^{\dagger} \widehat{a}\right\rangle \pm\left\langle a \wedge^{2}\right\rangle \pm\left\langle a \wedge^{\dagger 2}\right\rangle .
$$

Therefore, with the help of Eq. (21), one can readily establish that

$$
\begin{aligned}
& \left\langle a \wedge^{\dagger} \hat{a}\right\rangle=\frac{\gamma_{c}}{k}\left[(\nu-\gamma)^{2}\left[\left\langle\widehat{N}_{a}\right\rangle+\left\langle\widehat{N}_{b}\right\rangle+4 \varepsilon\left\langle\widehat{m}_{c}\right\rangle\right]+\left(\frac{2 \xi}{\gamma_{c}}\right)^{2}\left[\left\langle\widehat{N}_{b}\right\rangle+\left\langle\widehat{N}_{c}\right\rangle\right]\right], \\
& \left\langle\widehat{a} a \wedge^{\dagger}\right\rangle=\frac{\gamma_{c}}{k}\left[(\nu-\gamma)^{2}\left[\left\langle\widehat{N}_{b}\right\rangle+\left\langle\widehat{N}_{c}\right\rangle+4 \varepsilon\left\langle\widehat{m}_{c}\right\rangle\right]+\left(\frac{2 \xi}{\gamma_{c}}\right)^{2}\left[\left\langle\widehat{N}_{a}\right\rangle+\left\langle\widehat{N}_{b}\right\rangle\right]\right], \\
& \left\langle a \wedge^{2}\right\rangle=\frac{\gamma_{c}}{k}\left[\left[(v-\gamma)^{2}+4 \xi^{2}\right]\left\langle\widehat{m}_{c}\right\rangle+\left(\frac{2 \xi}{\gamma_{c}}\right)^{2}\left[N+\left\langle\widehat{N}_{b}\right\rangle\right]\right]
\end{aligned}
$$

Furthermore, on account of Eqs. (42)-(44), the quadrature variances takes the form

$$
\begin{aligned}
\Delta a_{ \pm}^{2}= & \frac{\gamma_{c}}{k}\left[(\nu-\gamma)^{2}\left[N+\left\langle\widehat{N}_{b}\right\rangle+8 \varepsilon\left\langle\widehat{m}_{c}\right\rangle \pm 2\left\langle\widehat{m}_{c}\right\rangle\right]\right. \\
& \left.+\left(\frac{2 \xi}{\gamma_{c}}\right)^{2}\left[N+\left\langle\widehat{N}_{b}\right\rangle\right](1 \pm 2) \pm 8 \xi^{2}\left\langle\widehat{m}_{c}\right\rangle\right]
\end{aligned}
$$




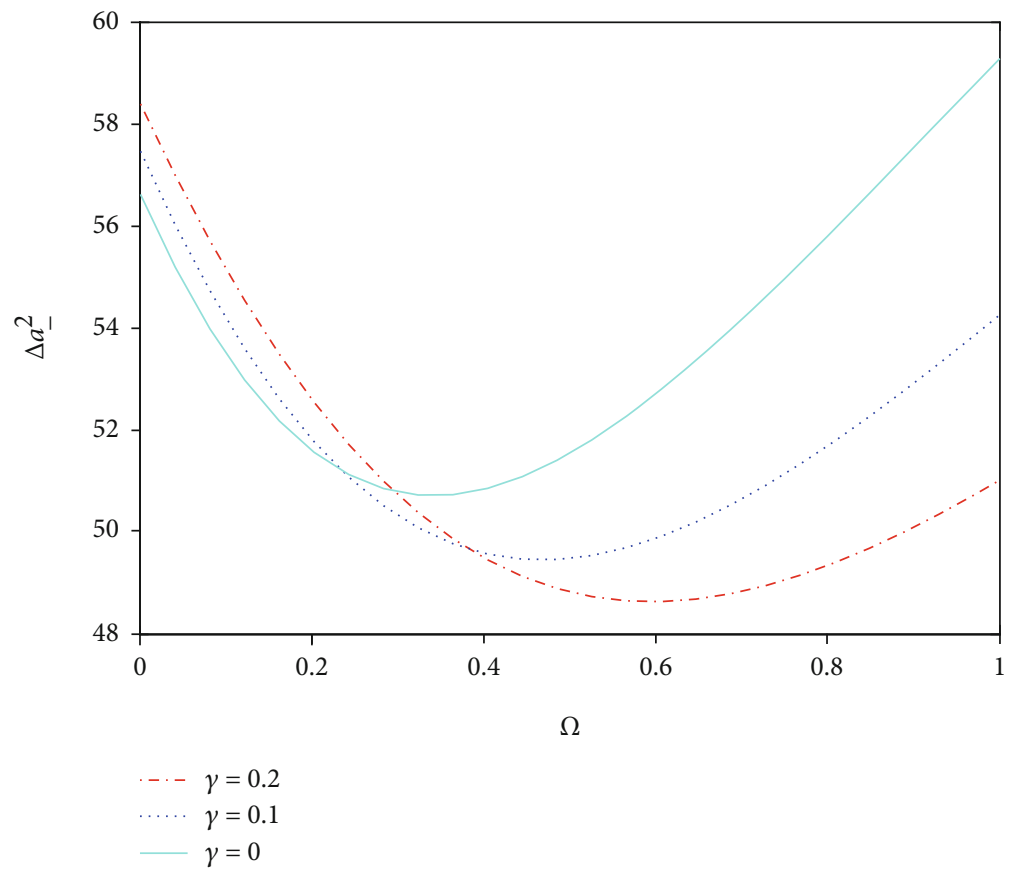

FIgURE 2: Plot of $\Delta a_{-}^{2}$ at steady state for Eq. (45) versus $\Omega$ for $\gamma_{c}=0.4, k=0.8, N=50$, and for different values of $\gamma$.

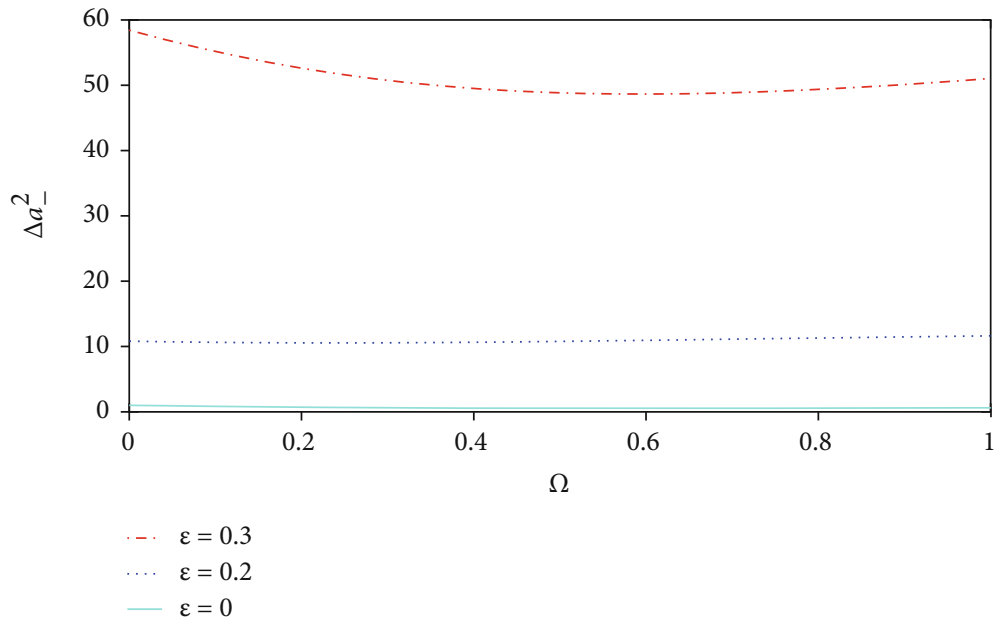

Figure 3: Plot of $\Delta a_{-}^{2}$ at steady state for [Eq. (45)] versus $\Omega$ for $\gamma_{c}=0.4, k=0.8, N=50$, and for different values of $\gamma$.

It is indicated in Figure 2 that squeezing exhibits in a different manner from a single cavity radiation, for values of $\Omega$ between 0 and 1, with differing degree of squeezing. Moreover, it is possible to realize that the degree of squeezing increases with the spontaneous emission decay constant, $\gamma$. Hence, one can observe that a light produced by a degenerate three-level laser with a parametric amplifier can exhibit different degrees of squeezing when, for example, compared to the light generated by a three-level laser in which the three-level atoms available in a closed cavity are pumped to the top level by means of electron bombardment $[24,25]$ as well as by coherent light $[7,8]$.

In Figure 3, we plot the intracavity quadrature variance of the cavity light vs. $\Omega$ for $\gamma=0.2, \gamma_{c}=0.4, \kappa=0.8, N=50$, and for different values of the amplitude of the pump mode that drives the NLC, $\varepsilon$. This figure indicates that the system under consideration exhibits a single-mode squeezing, and the degree of squeezing increases with the parameter $\varepsilon$. In this figure, the presence of parametric amplifier decreases the degree of squeezing. Furthermore, it is vividly presented in Figures 2 and 3 that the degree of squeezing is significantly degraded by the decreasing of spontaneous emission $(\gamma)$ and the increasing of the amplitude of the driving light on the parametric amplifier $(\varepsilon)$.

\section{The Mean Photon Number}

To learn about the brightness of the generated light, it is necessary to study the mean photon number of the cavity 


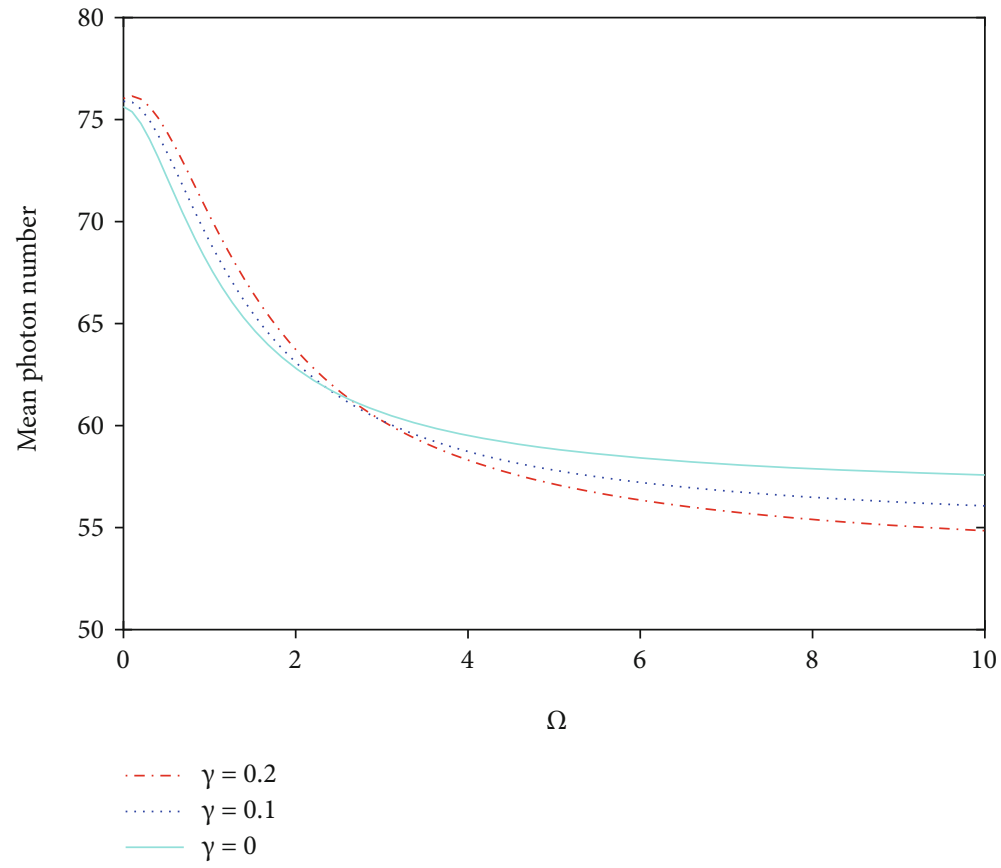

FIgURE 4: Plots of the steady-state mean photon number versus $\Omega$ for $\gamma_{c}=0.4, \kappa=0.8, N=50, \varepsilon=0.3$, and for different values of $\gamma$.

radiation that can be defined as

$$
\bar{n}=\left\langle a \wedge^{\dagger} \widehat{a}\right\rangle
$$

Hence, using Eq. (21) together with Eqs. (29) and (30), Eq. (46) takes the form

$$
\begin{aligned}
\bar{n}= & \frac{\gamma_{c} N}{k}\left\{(\nu-\gamma)^{2}\left[\frac{\left(2 \Gamma \nu+\zeta_{+}+8 \varepsilon v\right)(\xi+\Omega)}{4 v^{2}+2 \Gamma(2 \xi+\Omega)+(2 \Omega+\xi) \zeta_{+}}\right]\right. \\
& \left.+\left(\frac{2 \xi}{\gamma_{c}}\right)^{2}\left[1-\frac{\zeta_{+}(\xi+\Omega)}{4 v^{2}+2 \Gamma(2 \xi+\Omega)+(2 \Omega+\xi) \zeta_{+}}\right]\right\} .
\end{aligned}
$$

Next, we consider the case in which the nonlinear crystal is removed from the cavity. Thus, upon setting $\beta=0$ in Eq. (47), we get

$$
\bar{n}=\left(\frac{\gamma_{c}}{k} N\right)\left[\frac{\Omega^{2}\left(\gamma_{c}+\delta\right)}{\delta^{3}+\left(\gamma_{c}+2 \delta\right) \Omega^{2}}\right] .
$$
[7].

This result is the same as the result found by Abebe

In Figure 4, we plot the mean photon number of a cavity mode light versus $\Omega$ in the absence and presence of the spontaneous emission, $\gamma$. We observe from the plots in figure that the mean photon number of the cavity light beam is greater when $\gamma \neq 0$ than when $\gamma=0$ for $0 \leq \Omega \leq 2.3$. This implies that the mean photon number increases when $\gamma$ increases. It is easy to see from this figure that the presence of the spontaneous emission increases the mean photon number in region where there is strong squeezing. Hence, this system generates a bright and highly squeezed light.
In Figure 5, we plot the stead-state mean photon number versus $\Omega$ for $\gamma_{c}=0.4, \kappa=0.8, N=50, \gamma=0.2$, and for different values of $\varepsilon$. When we see these plots that the mean photon number increases as the parametric amplifier increases for $\Omega$ is small. Hence, the presence of parametric amplifier enhances the brightness of the cavity light.

\section{The $Q$ Function}

We now consider a two-mode cavity light beams represented by the operators $\hat{a}$ and $a \wedge^{\dagger}$ subject to the commutation relations

$$
\left[\widehat{a}, a \wedge^{\dagger}\right]=\lambda
$$

where

$$
\lambda=\frac{\gamma_{c}}{k}\left[\frac{\kappa^{2}}{\kappa^{2}-4 \varepsilon^{2}}\right]\left[\left\langle\widehat{N}_{c}\right\rangle-\left\langle\widehat{N}_{a}\right\rangle\right]
$$

is a constant $c$ number. Then, the $Q$ function for a cavity light is expressible as [24]

$$
Q\left(\alpha^{*}, \alpha\right)=\frac{\lambda}{\pi^{2}} \int d^{2} z \phi_{a}\left(z^{*}, z\right) \exp \left[z^{*} \alpha-z \alpha^{*}\right]
$$

in which

$$
\phi_{a}\left(z^{*}, z\right)=\operatorname{Tr}\left(\widehat{\rho} e^{-z^{*} a \wedge(t)} e^{z a \Lambda^{\dagger}(t)}\right)
$$

is the antinormally ordered characteristic function. Using the identity 


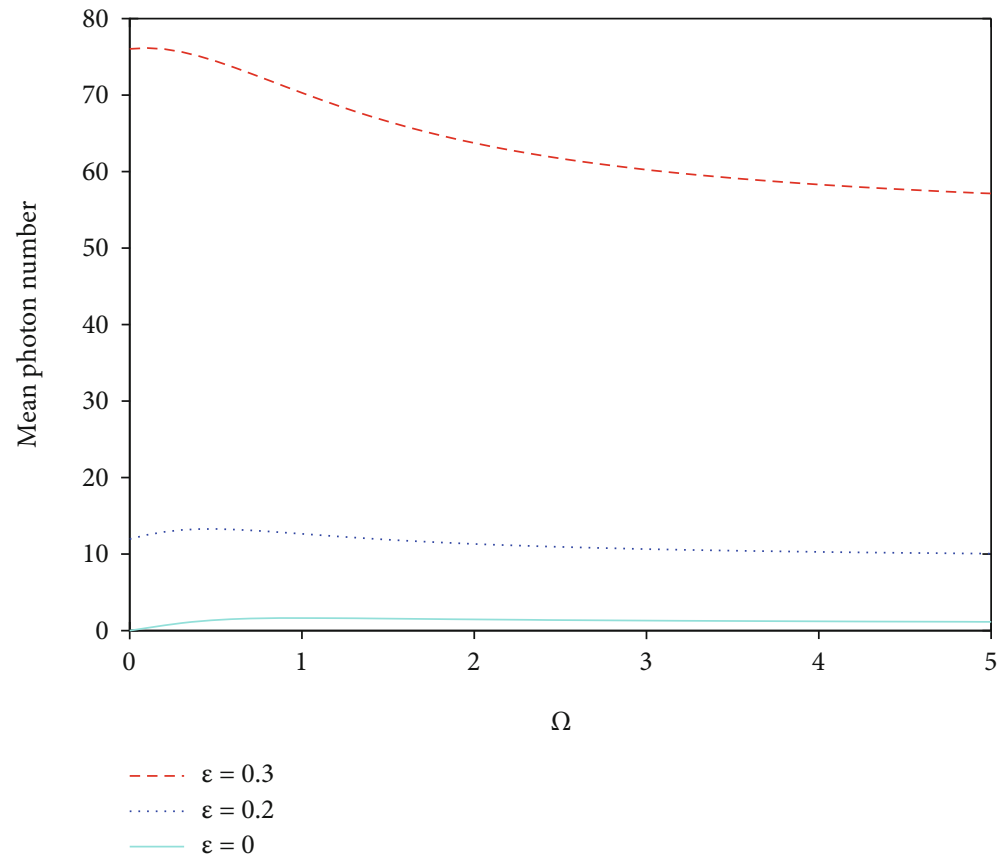

Figure 5: Plots of the steady-state mean photon number versus $\Omega$, for $\gamma_{c}=0.4, \kappa=0.8, N=50, \gamma=0.2$, and for different values of $\varepsilon$.

$$
e^{A \wedge} e^{B \wedge}=e^{A \wedge+B \wedge+\frac{1}{2}[A \wedge, B \wedge]}
$$

along with Eqs. (49), one can put Eq. (52) in the form

$$
\phi_{a}\left(z^{*}, z\right)=e^{-\frac{\lambda}{2} z^{*} z}\left\langle e^{\left[z a \wedge^{\dagger}(t)-z^{*} a \wedge(t)\right]}\right\rangle
$$

Since $\widehat{a}(t)$ is a Gaussian variable with zero mean, we can rewrite Eq. (54) as

$$
\begin{aligned}
\phi_{a}\left(z^{*}, z\right)= & \exp \left(-\frac{z^{*} z}{2}\left[\lambda+\left\langle a \wedge^{\dagger}(t) \hat{a}(t)\right\rangle+\left\langle\hat{a}(t) a \wedge^{\dagger}(t)\right\rangle\right]\right. \\
& \left.+\frac{z^{2}}{2}\left\langle a \wedge^{\dagger 2}(t)\right\rangle+\frac{z^{* 2}}{2}\left\langle a \wedge^{2}(t)\right\rangle\right) .
\end{aligned}
$$

On account of Eqs. (42)-(44), and (50), we see that

$$
\phi_{a}\left(z^{*}, z\right)=\exp \left[\frac{-a z^{*} z+b\left(z^{* 2}+z^{2}\right)}{2}\right]
$$

where

$$
\begin{aligned}
a= & \frac{\gamma_{c}}{k}\left\{\left(\frac{\nu-\gamma}{\gamma_{c}}\right)\left[\left\langle\widehat{N}_{c}\right\rangle-\left\langle\widehat{N}_{a}\right\rangle\right]+\left[\frac{2 \xi(\nu-\gamma)}{\gamma_{c}^{2}}\right]\left\langle\widehat{m}_{c}\right\rangle\right. \\
& \left.+\left[\frac{(\nu-\gamma)^{2}+2\left(\zeta_{+}-\Omega\right)}{\gamma_{c}^{2}}\right]\left[N+\left\langle\widehat{N}_{b}\right\rangle\right]\right\},
\end{aligned}
$$

$$
\begin{aligned}
b= & \frac{\gamma_{c}}{k}\left\{\left[\frac{2(\nu-\gamma)\left(\zeta_{+}-\Omega\right)}{\gamma_{c}^{2}}\right]\left[N+\left\langle\widehat{N}_{b}\right\rangle\right]\right. \\
& \left.+\left[\frac{(\nu-\gamma)^{2}+2\left(\zeta_{+}-\Omega\right)}{\gamma_{c}^{2}}\right]\left\langle\widehat{m}_{c}\right\rangle\right\} .
\end{aligned}
$$

Now, by substituting Eq. (56) into Eq. (51), we have

$$
Q\left(\alpha^{*}, \alpha\right)=\frac{\lambda}{\pi^{2}} \int d^{2} z \exp \left[-a z^{*} z+z^{*} \alpha-z \alpha^{*}+b\left[\frac{z^{* 2}}{2}+\frac{z^{2}}{2}\right]\right.
$$

Carrying out the integration using the relation [24].

$$
\begin{aligned}
& \int \frac{d^{2} z}{\pi} e^{-a z^{*} z+b z+c z^{*}+A z^{2}+B z^{* 2}} \\
& =\left[\frac{1}{a^{2}-4 A B}\right]^{2} \times \exp \left[\frac{a b c+A c^{2}+B b^{2}}{a^{2}-4 A B}\right], a>0,
\end{aligned}
$$

one can easily verify that

$$
Q\left(\alpha^{*}, \alpha, t\right)=\frac{\chi}{\pi} \exp \left[-p \alpha^{*} \alpha+q\left(\frac{\alpha^{* 2}}{2}+\frac{\alpha^{2}}{2}\right)\right]
$$

where $\chi=\lambda / \sqrt{a^{2}-b^{2}}, p=a /\left(a^{2}-b^{2}\right)$, and $q=\mathrm{b} /\left(a^{2}-b^{2}\right)$. 


\section{The Photon Number Distribution}

The photon number distribution for a single-mode light defined by

$$
P(n, t)=\left\langle n\left|\widehat{\rho}\left(a \wedge^{\dagger}, \widehat{a}\right)\right| n\right\rangle
$$

is expressible in terms of the $Q$ function as $[17,24,26]$.

$$
P(n, t)=\frac{\pi}{n !} \frac{\partial^{2 n}}{\partial \alpha^{* n} \partial \alpha^{n}}\left[Q\left(\alpha^{*}, \alpha, t\right) \exp \left[\alpha^{*} \alpha\right]\right]_{\alpha^{*}=\alpha=0} .
$$

On account of Eq. (61), we see that

$$
\begin{aligned}
P(n, t)= & \frac{\lambda}{n !}\left(p^{2}-q^{2}\right)^{1 / 2} \frac{\partial^{2 n}}{\partial \alpha^{* n} \partial \alpha^{n}} \times \exp \\
& \left.\cdot\left[(1-p) \alpha^{*} \alpha+q\left(\frac{\alpha^{* 2}}{2}+\frac{\alpha^{2}}{2}\right)\right]\right|_{\alpha^{*}=\alpha=0}
\end{aligned}
$$

Moreover, on expanding the exponential in power series

$$
\begin{aligned}
e^{(1-p) \alpha^{*} \alpha} & =\sum_{k}^{\infty} \frac{(1-p)^{k}}{k !} \alpha^{* k} \alpha^{k}, \\
e^{\frac{q \alpha^{* 2}}{2}} & =\sum_{l}^{\infty} \frac{q^{l}}{2^{l} l !} \alpha^{* 2 l}, \\
e^{\frac{q^{*} \alpha^{2}}{2}} & =\sum_{m}^{\infty} \frac{q^{* m}}{2^{m} m !} \alpha^{2 m},
\end{aligned}
$$

expression (64) can be put in the form

$$
P(n, t)=\frac{\lambda}{n !}\left(p^{2}-q^{2}\right)^{1 / 2} \sum_{k, l, m} \frac{(1-p)^{k}}{k !} \frac{q^{* l}}{l !} \frac{q^{m}}{m !} \frac{\partial^{n}}{\partial \alpha^{n}} \alpha^{k+2 l} \times\left.\frac{\partial^{n}}{\partial \alpha^{* n}} \alpha^{* k+2 m}\right|_{\alpha^{*}=\alpha=0}
$$

Upon carrying out the differentiation with the help of the relation

$$
\frac{\partial^{m}}{\partial x^{n}}=\frac{n !}{(n-m) !} \delta_{n, m}
$$

we get

$$
\begin{aligned}
P(n, t)= & \frac{\lambda}{n !}\left(p^{2}-q^{2}\right)^{1 / 2} \sum_{k, l, m} \frac{(1-p)^{k}}{k !} \frac{q^{* l}}{l !} \frac{q^{m}}{m !} \\
& \times \frac{(k+2 l) !}{(k+2 l-n) !} \frac{(k+2 m) !}{(k+2 m-n) !} \times \delta_{k+2 l, n} \delta_{k+2 m, n}
\end{aligned}
$$

Now, applying the property of the Kronecker delta, $l=m=(n-k) / 2$, the photon number distribution for the signal mode can be written as

$$
P(n, t)=\lambda\left(p^{2}-q^{2}\right)^{1 / 2} \sum_{k=0}^{n} n ! \frac{(1-p)^{k} q^{*(n-k / 2)} q^{(n-k / 2)}}{(k !)^{2}[(n-k / 2) !]^{2}},
$$

where $[n]=n / 2$ for even $n$ and $[n]=(n-1) / 2$ for odd $n$ [26]. From this result, we note that there is a finite probability of finding odd number of signal photons. This is due to the fact that, although the signal photons are generated in pairs, there is a possibility for an odd number of signal photons to leave the cavity via the port mirror. This must be then the reason for the possibility to observe an odd number of signal photons inside the cavity.

\section{Conclusion}

In this paper, we have studied the squeezing and statistical properties of the light produced by the coherently driven degenerate three-level laser with an open cavity and coupled to a two-mode vacuum reservoir via a single-port mirror. We have carried out our analysis by putting the vacuum noise operators in normal order and applying the large-time approximation scheme. Applying the solutions of the equations of evolution for the expectation values of the atomic operators and the quantum Langevin equations for the cavity mode operators, we have determined the mean photon number and the quadrature squeezing.

We have found that the light generated by the three-level laser is in a squeezed state, and the squeezing occurs in the minus quadrature. We have seen that the presence of spontaneous emission increases the degree of squeezing of the cavity light. On the other hand, the brightness of the cavity light enhanced in the presence of both the spontaneous emission and the parametric amplifier. The maximum mean photon number occurs for both cases at $\Omega$ near to zero. Finally, we have calculated with the aid of the $Q$ function, the photon number distribution for the cavity light beams. Therefore, there is a finite probability of finding odd number of signal photons. This is due to the fact that, although the signal photons are generated in pairs, there is a possibility for an odd number of photons to leave the cavity through the port mirror

\section{Appendix}

\section{A.1. Equations of Evolution of the Atomic Operators}

Here, we seek to obtain the equations of evolution of the expectation values of the atomic operators by applying the master equation and the large-time approximation scheme. To this end, making use of the master equation described by Eq. (8) for any operator $\widehat{A}$ and the fact that

$$
\frac{d}{d t}\langle\widehat{A}\rangle=\operatorname{Tr}\left(\frac{d \widehat{\rho}(t)}{d t} \widehat{A}\right)
$$


it is not difficult to verify that

$$
\begin{aligned}
& \frac{d}{d t}\left\langle\widehat{\sigma}_{a}^{k}\right\rangle=g \operatorname{Tr}\left[\widehat{\sigma}_{a}^{\dagger k} \widehat{a} \widehat{\rho} \widehat{\sigma}_{a}^{k}-a \wedge^{\dagger} \widehat{\sigma}_{a}^{k} \widehat{\rho} \widehat{\sigma}_{a}^{k}+\widehat{\sigma}_{b}^{\dagger k} \widehat{a} \hat{\rho} \widehat{\sigma}_{a}^{k}\right. \\
& -a \wedge^{\dagger} \widehat{\sigma}_{b}^{k} \widehat{\rho} \widehat{\sigma}_{a}^{k}-\widehat{\rho} \widehat{\sigma}_{a}^{\dagger k} \widehat{a} \widehat{\sigma}_{a}^{k}+\widehat{\rho} a \wedge^{\dagger} \widehat{\sigma}_{a}^{k} \widehat{\sigma}_{a}^{k} \\
& \left.-\widehat{\rho} \widehat{\sigma}_{b}^{\dagger k} \widehat{a}_{\sigma_{a}}^{k}+\widehat{\rho} a \wedge^{\dagger} \widehat{\sigma}_{b}^{k} \widehat{\sigma}_{a}^{k}\right] \\
& +\frac{\gamma}{2} \operatorname{Tr}\left[2 \widehat{\sigma}_{a}^{k} \widehat{\rho} \widehat{\sigma}_{a}^{\dagger k} \widehat{\sigma}_{a}^{k}-\widehat{\sigma}_{a}^{\dagger k} \widehat{\sigma}_{a}^{k} \widehat{\rho} \widehat{\sigma}_{a}^{k}-\widehat{\rho} \widehat{\sigma}_{a}^{\dagger k} \widehat{\sigma}_{a}^{k} \widehat{\sigma}_{a}^{k}\right] \\
& +\frac{\gamma}{2} \operatorname{Tr}\left[2 \widehat{\sigma}_{b}^{k} \widehat{\rho} \widehat{\sigma}_{b}^{\dagger k} \widehat{\sigma}_{a}^{k}-\widehat{\sigma}_{b}^{\dagger k} \widehat{\sigma}_{b}^{k} \widehat{\rho} \widehat{\sigma}_{a}^{k}-\widehat{\rho} \widehat{\sigma}_{b}^{\dagger k} \widehat{\sigma}_{b}^{k} \widehat{\sigma}_{a}^{k}\right] \\
& +\frac{\varepsilon}{2} \operatorname{Tr}\left[a \wedge^{2} \widehat{\rho} \widehat{\sigma}_{a}^{k}-\widehat{\rho} a \wedge^{2} \widehat{\sigma}_{a}^{k}+\widehat{\rho} a \wedge^{\dagger 2} \widehat{\sigma}_{a}^{k}-a \wedge^{\dagger 2} \widehat{\rho} \widehat{\sigma}_{a}^{k}\right] \\
& +\frac{\Omega}{2} \operatorname{Tr}\left[\widehat{\sigma}_{c}^{\dagger k} \hat{\rho} \widehat{\sigma}_{a}^{k}-\widehat{\sigma}_{c}^{k} \widehat{\rho} \widehat{\sigma}_{a}^{k}+\widehat{\rho} \widehat{\sigma}_{c}^{\dagger k} \widehat{\sigma}_{a}^{k}-\widehat{\rho} \widehat{\sigma}_{c}^{k} \widehat{\sigma}_{a}^{k}\right] .
\end{aligned}
$$

Now, applying the cyclic property of the trace operation on the above equation results in

$$
g \operatorname{Tr}\left(\hat{\rho} \widehat{\sigma}_{a}^{k} \widehat{\sigma}_{a}^{\dagger k} \widehat{a}\right)=g\left\langle\widehat{\eta}_{b}^{k} \widehat{a}\right\rangle
$$

Following the same procedure on the remaining terms, one can readily establish that

$$
\frac{d}{d t}\left\langle\widehat{\sigma}_{a}^{k}\right\rangle=g\left(\left\langle\hat{\eta}_{b}^{k} \hat{a}\right\rangle-\left\langle\hat{\eta}_{a}^{k} \hat{a}\right\rangle+\left\langle a \wedge^{\dagger} \widehat{\sigma}_{c}^{k}\right\rangle\right)+\frac{\Omega}{2}\left\langle\widehat{\sigma}_{b}^{\dagger k}\right\rangle-\gamma\left\langle\widehat{\sigma}_{a}^{k}\right\rangle,
$$

$$
\frac{d}{d t}\left\langle\widehat{\sigma}_{b}^{k}\right\rangle=g\left(\left\langle\hat{\eta}_{c}^{k} \hat{a}\right\rangle-\left\langle a \Lambda^{\dagger} \widehat{\sigma}_{c}^{k}\right\rangle-\left\langle\hat{\eta}_{b}^{k} \hat{a}\right\rangle\right)-\frac{\Omega}{2}\left\langle\widehat{\sigma}_{a}^{\dagger k}\right\rangle-\frac{\gamma}{2}\left\langle\widehat{\sigma}_{a}^{k}\right\rangle,
$$

$$
\frac{d}{d t}\left\langle\widehat{\sigma}_{c}^{k}\right\rangle=g\left(\left\langle\widehat{\sigma}_{b}^{k} \hat{a}\right\rangle-\left\langle\widehat{\sigma}_{a}^{k} \hat{a}\right\rangle\right)+\frac{\Omega}{2}\left[\left\langle\hat{\eta}_{c}^{k}\right\rangle-\left\langle\hat{\eta}_{a}^{k}\right\rangle\right]-\frac{\gamma}{2}\left\langle\widehat{\sigma}_{c}^{k}\right\rangle,
$$

$\frac{d}{d t}\left\langle\widehat{\eta}_{a}^{k}\right\rangle=g\left(\left\langle\hat{\sigma}_{a}^{\dagger k} \hat{a}\right\rangle+\left\langle a \wedge^{\dagger} \widehat{\sigma}_{a}^{k}\right\rangle\right)+\frac{\Omega}{2}\left[\left\langle\widehat{\sigma}_{c}^{k}\right\rangle+\left\langle\widehat{\sigma}_{c}^{\dagger k}\right\rangle\right]-\gamma\left\langle\widehat{\eta}_{a}^{k}\right\rangle$,

$\frac{d}{d t}\left\langle\hat{\eta}_{b}^{k}\right\rangle=g\left(\left\langle\widehat{\sigma}_{b}^{\dagger} \widehat{a}\right\rangle-\left\langle\widehat{\sigma}_{a}^{\dagger k} \hat{a}\right\rangle-\left\langle a \Lambda^{\dagger} \widehat{\sigma}_{a}^{k}\right\rangle+\left\langle a \Lambda^{\dagger} \widehat{\sigma}_{b}^{k}\right\rangle\right)-\gamma\left\langle\widehat{\eta}_{b}^{k}\right\rangle$,

where $\widehat{\eta}_{a}^{k}=|a\rangle_{k k}\left\langle a\left|, \widehat{\eta}_{b}^{k}=\right| b\right\rangle_{k k}\langle b|$, and $\widehat{\eta}_{c}^{k}=|c\rangle_{k k}\langle c|$ are the probabilities of the atoms on the top, middle, and bottom levels, respectively.

Now, introducing Eq. (19) into Eqs. (A.4)-(A.8) and sum over the $N$ three-level atoms, we get

$$
\begin{aligned}
& \frac{d}{d t}\left\langle\widehat{m}_{a}\right\rangle=-v\left\langle\widehat{m}_{a}\right\rangle-\frac{1}{2} \zeta_{-}\left\langle\widehat{m}_{b}^{\dagger}\right\rangle, \\
& \frac{d}{d t}\left\langle\widehat{m}_{b}\right\rangle=-\frac{v}{2}\left\langle\widehat{m}_{b}\right\rangle-\frac{1}{2} \zeta_{+}\left\langle\widehat{m}_{a}^{\dagger}\right\rangle,
\end{aligned}
$$

$$
\frac{d}{d t}\left\langle\widehat{m}_{c}\right\rangle=-\frac{v}{2}\left\langle\widehat{m}_{c}\right\rangle+\xi\left[\left\langle\widehat{N}_{c}\right\rangle-\left\langle\widehat{N}_{b}\right\rangle\right]+\frac{\Omega}{2}\left[\left\langle\widehat{N}_{c}\right\rangle-\left\langle\widehat{N}_{a}\right\rangle\right],
$$

$$
\begin{aligned}
\frac{d}{d t}\left\langle\widehat{N}_{a}\right\rangle & =-v\left\langle\widehat{N}_{a}\right\rangle+\frac{1}{2} \zeta_{+}\left[\left\langle\widehat{m}_{c}\right\rangle+\left\langle\widehat{m}_{c}^{\dagger}\right\rangle\right], \\
\frac{d}{d t}\left\langle\widehat{N}_{b}\right\rangle & =-v\left\langle\widehat{N}_{b}\right\rangle-\xi\left[\left\langle\widehat{m}_{c}\right\rangle+\left\langle\widehat{m}_{c}^{\dagger}\right\rangle\right]+[v-\gamma]\left\langle\widehat{N}_{a}\right\rangle,
\end{aligned}
$$

where $\gamma_{c}=4 g^{2} / \kappa$ is the stimulated emission decay constant, whereas

$$
\begin{aligned}
\nu & =\gamma+\frac{\gamma_{c} \kappa^{2}}{\kappa^{2}-4 \varepsilon^{2}}, \\
\zeta_{ \pm} & =\frac{2 \gamma_{c} \varepsilon \kappa}{\kappa^{2}-4 \varepsilon^{2}} \pm \Omega, \\
\xi & =\frac{\gamma_{c} \varepsilon \kappa}{\kappa^{2}-4 \varepsilon^{2}} .
\end{aligned}
$$

For $N$ number of atoms, we see that $\widehat{m}_{j}=\sum_{k=1}^{N} \widehat{\sigma}_{j}^{k}$ and $\widehat{N}_{j}=\sum_{k=1}^{N} \widehat{\eta}_{j}^{k}$, (where $\left.j=a, b, c\right)$. Hence, the operators $\widehat{N}_{a}$, $\widehat{N}_{b}$, and $\widehat{N}_{c}$ represent the number of atoms in the top, middle, and bottom levels, respectively. In addition, employing the completeness relation

$$
\widehat{\eta}_{a}^{k}+\widehat{\eta}_{b}^{k}+\widehat{\eta}_{c}^{k}=\widehat{I}
$$

we easily arrive at

$$
\left\langle\widehat{N}_{a}\right\rangle+\left\langle\widehat{N}_{b}\right\rangle+\left\langle\widehat{N}_{c}\right\rangle=N
$$

Furthermore, using the definition $\widehat{\sigma}_{a}^{k}=|b\rangle_{k k}\langle a|$ and setting for any $k, \widehat{\sigma}_{a}^{k}=|b\rangle\langle a|$, we have $\widehat{m}_{a}=N|b\rangle\langle a|$. Following the same procedure, one can also easily establish that $\widehat{m}_{b}=$ $N|c\rangle\left\langle b\left|, \widehat{m}_{c}=N\right| c\right\rangle\left\langle a\left|, \widehat{N}_{a}=N\right| a\right\rangle\left\langle a\left|, \widehat{N}_{b}=N\right| b\right\rangle\langle b|$, and $\widehat{N}_{c}$ $=N|c\rangle\left\langle c\right.$. Using the definition $\widehat{m}=\widehat{m}_{a}+\widehat{m}_{b}$, [27] it can be readily established that

$$
\left\{\begin{array}{l}
m \wedge^{\dagger} \widehat{m}=N\left(\widehat{N}_{a}+\widehat{N}_{b}\right) \\
\widehat{m} m \wedge^{\dagger}=N\left(\widehat{N}_{b}+\widehat{N}_{c}\right) \\
m \wedge^{2}=N \widehat{m}_{c} .
\end{array}\right.
$$

\section{Data Availability}

The data used to findings the study are included within the article.

\section{Conflicts of Interest}

The authors declare that there is no conflict of interest regarding the publication of this paper. 


\section{Acknowledgments}

It is our pleasure to thank Jimma University, College of Natural Sciences Research and Postgraduate Coordination Office for all they did for us, their help, encouragement, and financial support during our research.

\section{References}

[1] C. W. Gardiner, "Inhibition of atomic phase decays by squeezed light: a direct effect of squeezing," Physical Review Letters, vol. 56, no. 18, pp. 1917-1920, 1986.

[2] S. Qamar, S. Qamar, and M. S. Zubairy, "Effect of phase fluctuations on entanglement generation in a correlated emission laser with injected coherence," Optics communications, vol. 283, no. 5, pp. 781-785, 2010.

[3] T. Abebe, "Enhancement of squeezing and entanglement in a non-degenerate three-level cascade laser with coherently driven cavity," Ukrainian Journal of Physics, vol. 63, no. 8, pp. 733-739, 2018.

[4] E. Alebachew, "Enhanced squeezing and entanglement in a non-degenerate three-level cascade laser with injected squeezed light," Optics Communication, vol. 280, no. 1, pp. 133-141, 2007.

[5] S. Tesfa, "Effect of dephasing on quantum features of the cavity radiation of an externally pumped correlated emission laser," Physical Review A, vol. 79, no. 6, article 063815, 2009.

[6] N. A. Ansari, J. G. Banacloche, and M. S. Zubairy, "Phase-sensitive amplification in a three-level atomic system," Physical Review A, vol. 41, no. 9, pp. 5179-5186, 1990.

[7] T. Abebe, "The quantum analysis of nondegenerate three-level laser with spontaneous emission and noiseless vacuum reservoir," Ukrainian Journal of Physics, vol. 63, no. 11, pp. 969978, 2018.

[8] T. Abebe, "Coherently driven nondegenerate three-level laser with noiseless vacuum reservoir," Bulgarian Journal of Physics, vol. 45, no. 4, pp. 357-373, 2018.

[9] H. Xiong, M. O. Scully, and M. S. Zubairy, "Correlated spontaneous emission laser as an entanglement amplifier," Physical Review Letters, vol. 94, no. 2, article 023601, 2005.

[10] C. Gashu, E. Mosisa, and T. Abebe, "Entanglement quantification of correlated photons generated by three-level laser with parametric amplifier and coupled to a two-mode vacuum reservoir," Advances in Mathematical Physics, vol. 2020, 14 pages, 2020.

[11] C. Gashu and T. Abebe, "Externally induced entanglement amplification in a coherently pumped emission of laser with parametric amplifier and coupled to squeezed vacuum reservoir," Physica Scripta, vol. 95, no. 7, p. 075105, 2020.

[12] T. Abebe, N. Gemechu, C. Gashu, K. Shogile, S. Hailemariam, and S. Adisu, "The quantum analysis of nonlinear optical parametric processes with thermal reservoirs," International Journal of Optics, vol. 2020, 11 pages, 2020.

[13] T. Abebe, N. Gemechu, K. Shogile, S. Hailemariam, C. Gashu, and S. Adisu, "Entanglement quantification using various inseparability criteria for correlated photons," Romanian Journal of Physics, vol. 65, no. 3-4, p. 107, 2020.

[14] J. Anwar and M. S. Zubairy, "Quantum-statistical properties of noise in a phase-sensitive linear amplifier," Physical Review A, vol. 49, no. 1, pp. 481-484, 1994.
[15] C. A. Blockley and D. F. Walls, "Intensity fluctuations in a frequency down-conversion process with three-level atoms," Physical Review A, vol. 43, no. 9, pp. 5049-5056, 1991.

[16] N. Lu, F. X. Zhao, and J. Bergou, "Nonlinear theory of a twophoton correlated-spontaneous-emission laser: a coherently pumped two-level-two-photon laser," Physical Review A, vol. 39, no. 10, pp. 5189-5208, 1989.

[17] B. Daniel and K. Fesseha, "The propagator formulation of the degenerate parametric oscillator," Optics Communication, vol. 151, no. 4-6, pp. 384-394, 1998.

[18] F. Kassahun, Fundamental of Quantum Optics, Lulu, North Carolina, 2008.

[19] S. Tesfa, "Continuous variable entanglement in a coherently pumped correlated emission laser," Journal of Physics B: Atomic, Molecular and Optical Physics, vol. 41, no. 5, article 055503, 2008.

[20] G. New, Introduction to Nonlinear Optics, Cambridge University Press, New York, 2011.

[21] W. H. Louisell, Quantum Statistical Properties of Radiation, Wiley, New York, 1973.

[22] E. Alebachew and K. Fesseha, "A degenerate three-level laser with a parametric amplifier," Optics Communication, vol. 265, no. 1, pp. 314-321, 2006.

[23] S. Tesfa, "Entanglement amplification in a nondegenerate three-level cascade laser," Physical Review A, vol. 74, no. 4, article 043816, 2006.

[24] F. Kassahun, Refined Quantum Analysis of Light, Create Space Independent Publishing Platform, United States, 2014.

[25] F. Kassahun, Three-Level Laser Dynamics with the Atoms Pumped by Electron Bombardment, 2012, https://arxiv.org/ abs/1105.1438v3.

[26] B. Teklu, "Parametric oscillation with the cavity mode driven by coherent light and coupled to a squeezed vacuum reservoir," Optics Communication, vol. 261, no. 2, pp. 310-321, 2006.

[27] F. Kassahun, "Stimulated emission by two-level atoms pumped to the upper level," Optics Communication, vol. 284, no. 5, pp. 1357-1363, 2011. 